\title{
Challenges in the Analysis of Gangliosides by LC-MS
}

\author{
Thomas Muggli, Claudia Bühr, and Stefan Schürch*
}

\begin{abstract}
Gangliosides are a family of conjugates consisting of a polar sialoglycan head group and a hydrophobic ceramide tail. Gangliosides are of high abundance in neuronal tissues and are involved in numerous biological processes, such as cell-cell recognition, adhesion, and signal transduction. Alteration of the ganglioside profile is associated with various neurodegenerative diseases and there is indication that gangliosides are involved in the pathogenesis of Parkinson's and Huntington's disease. The development of refined methods for the analysis of gangliosides by high-performance liquid chromatography coupled to mass spectrometry (HPLC-MS) has supported research with qualitative and quantitative data. However, the amphiphilic character of gangliosides renders their separation and mass spectrometric analysis challenging. In this article, the strengths of hydrophilic interaction liquid chromatography (HILIC) for baseline separation of gangliosides, including two structural isomers, and their structural characterization by tandem mass spectrometry are demonstrated. The importance of ion source parameter optimization is highlighted to prevent misleading ganglioside transformation due to in-source dissociation.
\end{abstract}

Keywords: Gangliosides · Glycosphingolipids · HILIC· In-source dissociation · Mass spectrometry

Thomas Muggli received his master's degree in chemistry and molecular sciences from the University of Bern in 2019. He completed his master thesis on the development of LC-MS based methods for the analysis of gangliosides in the group of Stefan Schürch. Currently, he is working towards his $\mathrm{PhD}$ in chemistry in the same group with his research focused on the development and application of ambient ionization techniques.

Claudia Bühr started her career as a chemical lab technician in the Institute of Biochemistry and Molecular Medicine at the University of Bern. She continued her education and received a diploma as chemical engineer in 1989 and started working in the Department of Pharma Research, Pharmacokinetics of F. Hoffmann-La Roche. In 1995 she joined the research laboratory of Visceral and Transplantation Surgery at the Inselspital Bern. Then she worked as a medical sales representative before she joined the mass spectrometry group of Stefan Schürch as a chemist in 2007.

Stefan Schürch received his diploma in chemistry and his $\mathrm{PhD}$ degree from the University of Bern, where he graduated with Urs Schlunegger in 1995. After a two-year postdoctoral stay with Charles L. Wilkins at the University of California, Riverside, he became leader of the mass spectrometry facility at the Department of Chemistry, Biochemistry and Pharmaceutical Sciences of the University of Bern, where he was appointed Associate Professor in 2017. Research of this group focuses on the characterization of biomolecule structures by mass spectrometry and on the elucidation of the processes involved in the dissociation of ions in the gas-phase.

\section{Structure and Biological Function of Gangliosides}

Gangliosides are major constituents of the neuronal cell surface, fulfilling key functions in membrane organization and cell signaling..$^{[1,2]}$ Based on their strong occurrence in ganglion cells, the German biochemist Ernst Klenk proposed in 1942 the term

${ }^{*}$ Correspondence: Prof. Dr. S. Schürch, E-Mail: stefan.schuerch@unibe.ch Departement für Chemie, Biochemie und Pharmazie,

University of Bern, Frieistrasse 3, $\mathrm{CH}-3012$ Bern 'gangliosides' for this kind of neuraminic acid containing lipids. ${ }^{[3]}$ Although they are omnipresent in tissues and body fluids, gangliosides are expressed predominantly in the nervous system, where they are involved in the formation of membrane domains, interact with membrane and extracellular proteins, and modulate receptor activity. ${ }^{[4]}$ Their functional importance was proven in experiments with genetically engineered mice, where the lack of ganglioside synthases caused drastically impaired nervous functions such as hearing loss and dysfunction in motor coordination, as well as demyelination and deterioration in the peripheral nervous system. ${ }^{[1]}$ Alteration of the ganglioside profile was found to promote the development of neurodegenerative diseases. ${ }^{[4]}$ Gangliosides are involved in an autoimmune response called Guillain-Barré syndrome, an acute paralytic neuropathy, or lysosomal storage disorders, such as the Tay-Sachs disease caused by excessive ganglioside accumulation, and further studies even suggest the involvement of gangliosides in Parkinson's and Huntington's disease. ${ }^{[1,4,5]}$ Due to their pivotal role in neurodegenerative diseases, gangliosides are also considered for therapeutic applications and serve as biomarkers for diagnostic purposes..$^{[4]}$

Gangliosides represent a sub-group of the glycosphingolipids (GSL) consisting of a sialic acid-containing polar head group connected to a hydrophobic ceramide part composed of the amino alcohol sphingosine and a fatty acid. More than 200 different ganglioside structures comprising a variety of headgroup and ceramide compositions have been discovered until today.[6] The ceramide tail is composed of a sphingosine connected to a fatty acid (often stearic acid) and anchors the molecule to the cell membrane. Huge variabilities of ceramide tail compositions are known, including different chain lengths and fatty acid saturation levels, especially among different species and types of tissues. The serine palmitoyltransferases which catalyze the acylation of serine with palmitoyl-CoA have a strong selectivity for $16 \pm 1$ carbon atoms containing fatty acyl-CoAs. This explains the dominating sphingosine variant with 18 carbons (2 from serine, 16 from palmitoyl-CoA). Nevertheless, in some animal tissues, such as mammalian brain, human gastrointestinal tract, and equine kidney, considerable amounts of $\mathrm{C} 20$-sphingoidbase were observed. ${ }^{[7]}$ The hydrophilic head group of gangliosides is based on $\beta-1-3$ - and $\beta-1$ - 
4-linked carbohydrates and mainly comprises glucose, galactose, $\mathrm{N}$-acetylgalactosamine, and sialic acids. Sialic acids are a family of nine-carbon sugars which mainly occurs in the ganglioside series found in adult mammals. More than 50 different sialic acids have been described till today, comprising numerous modifications, including $\mathrm{O}$-acetylation, $\mathrm{N}$-deacetylation, $\mathrm{O}$-methylation, sulphation, or lactonization. ${ }^{[8]}$ The major sialic acid found in healthy humans is 5-N-acetylneuraminic acid (Neu5Ac). [9]

The nomenclature of gangliosides follows the scheme proposed by Svennerholm, ${ }^{[10]}$ where the first letter represents the glycosphingolipid series, with $\mathrm{G}$ for the ganglio series. The second letter indicates the number of sialic acid moieties $(A=0, M=1, D=2, T=3$, and $Q=4)$, and the number at the third position is subtracted from five to yield the number of uncharged carbohydrate units present (Fig. 1). The letter at the last position refers to the biosynthetic pathway. For example, GD1a and GD1b originate from the biosynthetic a and b series, respectively, and both gangliosides comprise two sialic acid moieties along with four uncharged carbohydrates. They are structural isomers and only differ in the connection of the sialic acids to the ganglioside. GD1b contains a dimeric sialic acid unit, while GD1a carries two monomeric sialic acids.

Elucidating the crucial role gangliosides are playing in complex biological pathways requires sensitive and accurate analytical tools. Though liquid chromatography coupled to mass spectrometry is capable of providing qualitative and quantitative data, the fragile ganglioside structures require highly optimized analytical methods.

\section{Experimental}

Stock solutions of gangliosides GM1 (Carbosynth Ltd., United Kingdom), GD1a, GD1b, and GT1a (Hytest Ltd., Turku, Finland), were prepared in methanol at a concentration of $1 \mathrm{mg} / \mathrm{ml}$ and stored at $5{ }^{\circ} \mathrm{C}$. For analysis, the stock solutions were diluted to a final concentration of $25 \mu \mathrm{g} / \mathrm{ml}$ with a solvent mixture representing the starting chromatographic conditions.

\section{GMl ganglioside}
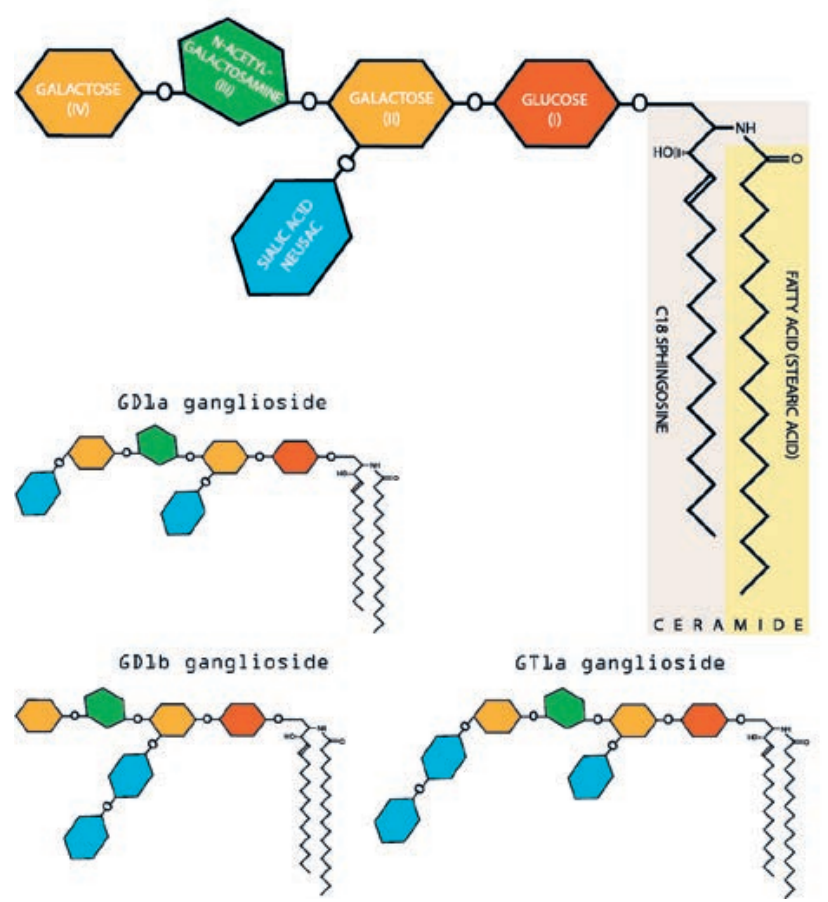

Fig. 1. Schematic representation of the four gangliosides discussed in this article. The hydrophobic ceramide tail is composed of a fatty acid bound to the amino alcohol sphingosine. The hydrophilic head group consists of a characteristic carbohydrate sequence bearing one or several sialic acids ( $\mathrm{N}$-acetylneuraminic acids).
The LC-MS method was developed on a Sciex ExionLC HPLC system coupled to a Sciex X500B high-resolution quadrupole timeof-flight mass spectrometer (AB Sciex, Baden, Switzerland). Liquid chromatography was performed on a Thermo Scientific Syncronis 100 $\times 2.1 \mathrm{~mm}$ HILIC column with a particle size of $5 \mu \mathrm{m}$ (Thermo Fisher Scientific, Reinach, Switzerland). A gradient starting at $90 \%$ acetonitrile $/ 10 \%$ water running to $65 \%$ acetonitrile $/ 35 \%$ water in 13 minutes was applied for separation of the gangliosides. Acetonitrile and water in LC/MS quality were obtained from Biosolve (Valkenswaard, The Netherlands). Both mobile phases were buffered to $\mathrm{pH} 6$ with $10 \mathrm{mM}$ ammonium acetate in LC-MS Ultra quality (Sigma-Aldrich, Buchs, Switzerland). The flow rate was set to $0.3 \mathrm{ml} / \mathrm{min}$ and the column oven temperature to $50{ }^{\circ} \mathrm{C}$. The autosampler injection volume was $10 \mu$ l. Silanized glass vials and inserts (BGB Analytik AG, Boeckten, Switzerland) were used in all experiments to effectively prevent sample adsorption on the vial surface.

Mass spectrometric analysis was based on electrospray ionization (ESI) operated in the negative ionization mode with the ion source heater gas temperature set to $375^{\circ} \mathrm{C}$ at 3.45 bar (50 psi) gas pressure and 3.45 bar ( 50 psi) nebulizer gas pressure. Fragmentation data of ganglioside mixtures were recorded in the information-dependent acquisition (IDA) mode with the collision energy settings in the range of $-30 \mathrm{~V}$ to $-50 \mathrm{~V}$ for the different gangliosides.

\section{Separation of Gangliosides}

The separation of regioselective ganglioside isomers on $\mathrm{C} 18$ columns remains difficult, ${ }^{[11-15]}$ as their separation is primarily determined by the length and the unsaturation degree of the hydrophobic tail group. ${ }^{[16,17]}$ Consequently, alternative chromatographic approaches with selectivity for more polar compounds are required for baseline separation of ganglioside structural isomers, such as GD1a and GD1b (Fig. 1).

Hydrophilic interaction liquid chromatography (HILIC) not only allows to retain highly polar molecules, it also confers a strong separation capability on them. Within the past two decades, the interest in HILIC-based analytical methods has strongly increased, ${ }^{[18]}$ as reflected by the considerable number of publications on the application of HILIC in pharmaceutical, biomedical, and clinical analysis, ${ }^{[19]}$ and HILIC plays an important role in the analysis of polar drugs in the growing field of metabolomics. [20,21] In recent years, HILIC in combination with mass spectrometry has found attention for the analysis of gangliosides. ${ }^{[22-26]}$

HILIC is a variant of normal phase chromatography in which the separation of analyte molecules is based on their partitioning between the mobile phase and a layer of water-enriched mobile phase partially immobilized on the polar stationary phase. ${ }^{[27]}$ The elution order of analytes in HILIC is inverse to reversed-phase chromatography, with faster elution of hydrophobic compounds and stronger retention of hydrophilic analytes. Consequently, HILIC provides the opportunity for separation of gangliosides according to the polarity of their hydrophilic head group. ${ }^{[28]}$

HILIC resulted in baseline separation of the four gangliosides including the structural isomers GD1a and GD1b (Fig. 2). HILIC demonstrated its excellent capability in separating gangliosides based on their hydrophilic head group, while putting aside the difference in hydrophobicity originating from different ceramide compositions. The longer $\mathrm{C} 20$ sphingosines eluted only a few seconds earlier compared to the shorter $\mathrm{C} 18$ sphingosines.

\section{Mass Spectrometry of Gangliosides}

Sialic acid-containing glycosphingolipids are preferentially analyzed as anions. The number of charges is influenced by the sialic acids of the head group, which results in the typically low charge states found for gangliosides. However, analysis by singlestage mass spectrometry alone does not provide unambiguous identification of the gangliosides, as isobaric ions (i.e. GD1a/ GD1b) cannot be differentiated from each other. 


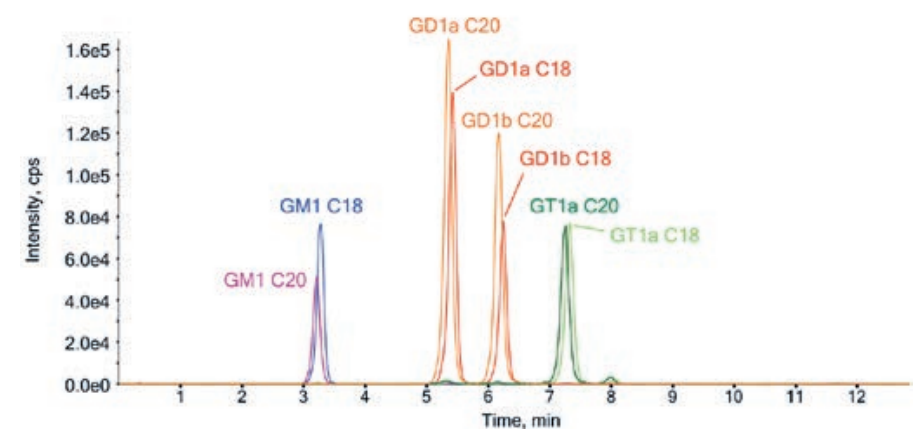

Fig. 2. Extracted ion current chromatogram demonstrating baseline separation of the four gangliosides GM1, GD1a, GD1b, and GT1a achieved by HILIC. The separation is mainly based on the interaction of the polar sialoglycan head groups with the stationary phase, while the C18/C20 sphingosine variants of each ganglioside caused slight shifts in the retention times only.

More extended structural information is obtained by detection of fragment ions generated by tandem mass spectrometric experiments. Collision-induced dissociation of glycosphingolipids in the gas-phase mainly results in scission of glycosidic bonds and the release of single or multiple sugar and sialic acid moieties, while the ceramide structure is less affected by dissociation. The sialic acid plays a key role in the collision-induced dissociation (CID) of gangliosides, as the negatively charged oxygen of the deprotonated carboxylic group acts as a nucleophile initiating substitution reactions. Attack of the alpha position in an intramolecular $\mathrm{S}_{\mathrm{N}} 2$ reaction forms an epoxide and simultaneously cleaves the glycosidic bond with the glycosidic oxygen remaining on the Y-ion, as illustrated by Fig. 3. ${ }^{[29]}$ The deprotonated hydroxy group of the newly formed Y-fragment ion can initiate further nucleophilic attacks and lead to secondary dissociation events.

Such dissociation is exemplified by the product ion spectra of doubly deprotonated GD1b and singly deprotonated GM1, both bearing the sialic acid(s) at the second sugar moiety (galactose (II)) and the ceramide with the C18 sphingosine (Fig. 4). Both product ions give evidence for the release of a sialic acid as the most prevalent fragmentation event, represented by the peaks of the $b_{1 \beta}$ ion at $m / z 290.0869$ and the $b_{2 \beta}$ ion at $m / z$ 290.0863, for GD1b and GM1, respectively. Another single sialic acid release is represented by the $c_{1 \beta}$ (GD1b) ion, which comprises the oxygen atom of the glycosidic bond. In contrast to GM1, GD1b exhibits a dimeric sialic acid, which is reflected by the peak of the $b_{2 b}$ fragment at $m / z$ 581.1819. The presence of the dimeric sialic acid
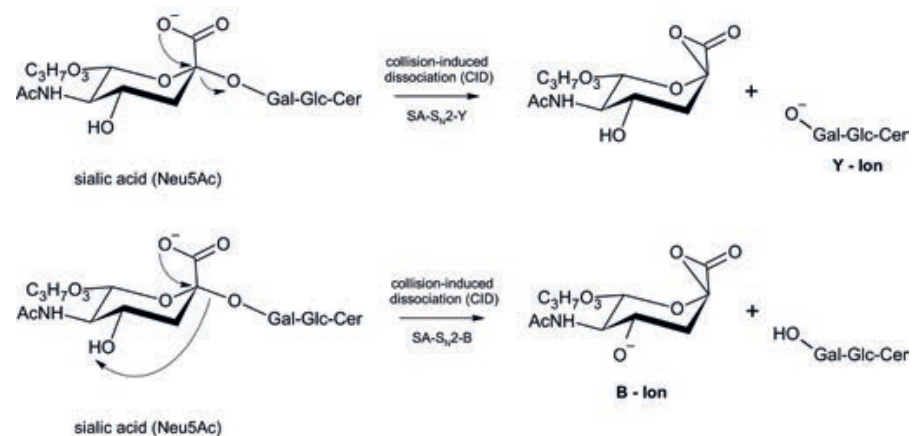

Fig. 3. The dissociation of gangliosides is initiated by the deprotonated carboxylic group of the sialic acid. The sialic acid is either released as a B-ion, or as a neutral with the charge remaining on the Gal-Glc-Cer structure. Release of the sialic acid occurs and results in the formation of a Y-ion which can undergo further dissociation steps. Figure adapted from ref. [29] fragment helps in distinguishing from gangliosides which contain monomeric sialic acids only. Though fragment ions may be of low abundance only, accurate mass analysis allows to unambiguously assign their origin. For example, GD1b shows the release of a Gal-GalNAc $b_{2}$ fragment ion $(m / z$ 364.1240), while cleavage of the corresponding glycosidic bond in GD1a results in the loss of the larger Neu5Ac-Gal-GalNAc unit as a c ion $(\mathrm{m} / \mathrm{z}$ 673.2309), which allows to distinguish GD1a from its isobaric GD1b sibling.

Remarkably, the GD1b product ion spectrum shows a peak at $m / z 1544.8695\left(\mathrm{y}_{1 \beta}\right)$, which originates from the loss of a sialic acid. This singly charged fragment ion is identical in mass and structure to the unfragmented GM1 precursor ion. Both spectra show the peak corresponding to the $\mathrm{C} 18$ sphingosine containing the ceramide linked to a glucose and a galactose unit $(\mathrm{m} / \mathrm{z} 888.6384$ for GM1 and $m / z 888.6399$ for GD1b), referred to as the $\mathrm{y}_{2 \beta} / \mathrm{y}_{2}$ fragment ion. The ceramide carrying only the glucose moiety is represented by the $\mathrm{y}_{1}$ and $\mathrm{z}_{1}$ ions $(\mathrm{m} / \mathrm{z}, 726.5879$ and $\mathrm{m} / \mathrm{z}, 708.5794$ for GM1; $m / z 726.5890$ and $m / z 708.5794$ for GD1b). Further loss of the remaining glucose moiety yields the plain ceramide as $\mathrm{y}_{0}$ ion at $m / z, 564.5349$. The $\mathrm{y}_{2 \beta}$ fragments $(\mathrm{m} / \mathrm{z}, 1253.7742$ for GM1 and $m / z, 1253.7744$ for GD1b) are the result of stripping-off all sialic acid moieties.
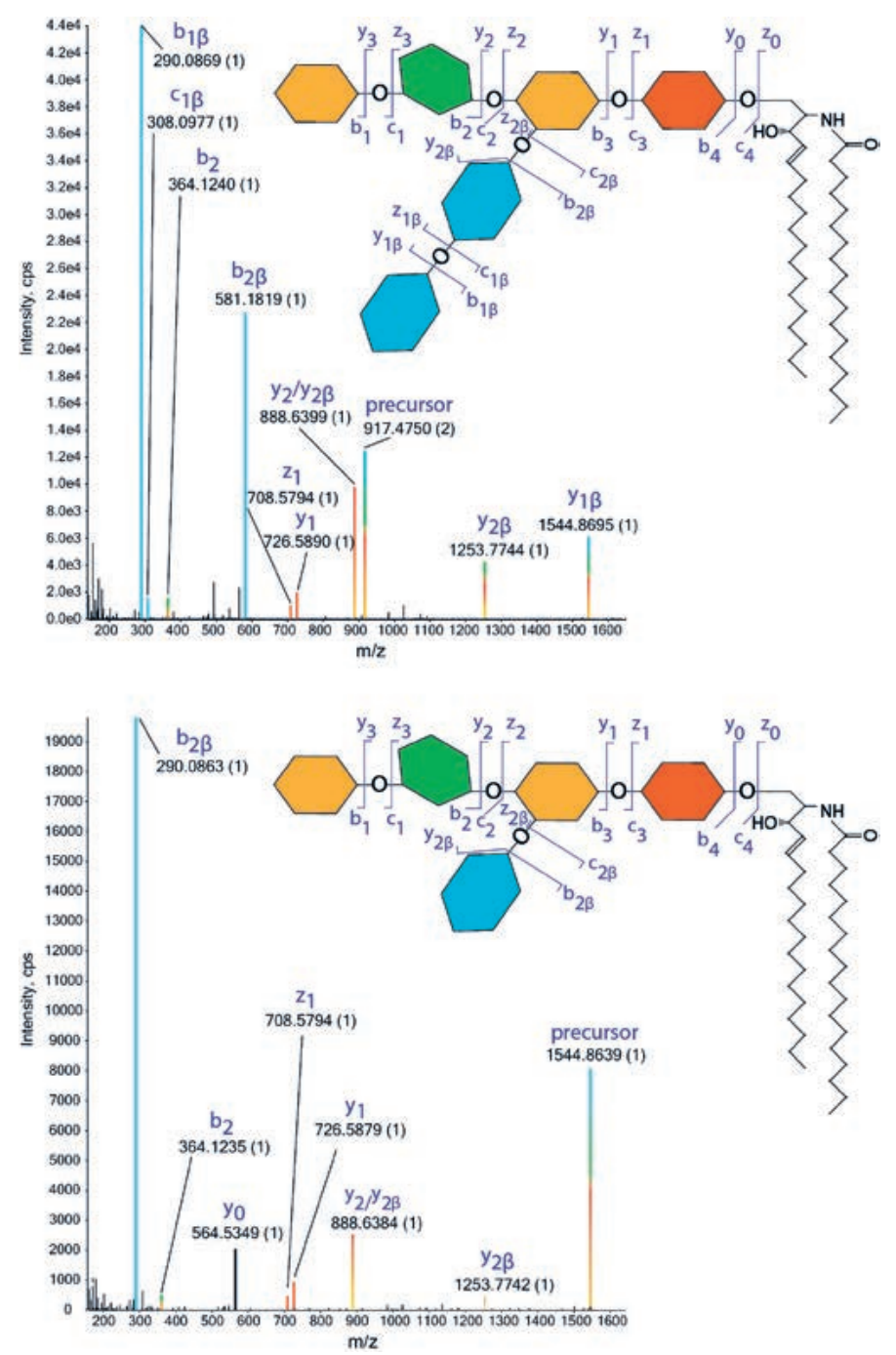

Fig. 4. Product ion spectra obtained by collision-induced dissociation of the doubly deprotonated GD1b (top) and singly deprotonated GM1 (bottom) gangliosides. Diagnostic fragment ions enable the elucidation of the sialoglycan pattern. Loss of a sialic acid from GD1b results in a fragment ion identical to GM1. The negative charge states of the ions are indicated in brackets. 
The ionization of gangliosides has to be performed under very soft ionization conditions, as these structures potentially undergo transformation into a different type of ganglioside, e.g. GD1a to GM1, GD1b to GM1, or GT1a to GD1 and GM1 as illustrated in Fig. 5. Despite the generally known soft characteristics of electrospray ionization, high electrospray source gas temperatures and elevated ion optic potentials increase the risk of unwanted in-source dissociation. Electrospray ionization is based on the evaporation of charged droplets in order to transfer the analytes into the gas phase. To assist in solvent evaporation, a heater gas is applied to the stream of electrosprayed droplets. Though higher gas temperatures were found to increase the yield of ganglioside ions while simultaneously reduce adduct formation, dissociation of labile gangliosides occurred at gas temperatures above $400{ }^{\circ} \mathrm{C}$.

Structural transformations originating from the loss of sialic acid moieties, which represent the preferred dissociation channel of gangliosides, are potentially occurring in the electrospray interface. Fig. 5 demonstrates the effect of the declustering potential DP (also referred to as cone voltage) on the stability of GT1a. This potential is applied to the electrospray interface to remove remaining solvent clusters from analyte ions after they entered the first vacuum stage. Inappropriate setting results either in loss of ion current or in dissociation of analyte ions due to collisions with residual gas. The mass spectrum recorded with an optimized DP of $-80 \mathrm{~V}$ shows the peaks of the doubly and triply charged ganglioside carrying the $\mathrm{C} 18$ and $\mathrm{C} 20$ sphingosine (Fig. 5, top). Increasing the DP leads to in-source dissociation and fragment ion peaks that mimic the presence of different gangliosides appear in the spectrum (Fig. 5, bottom).
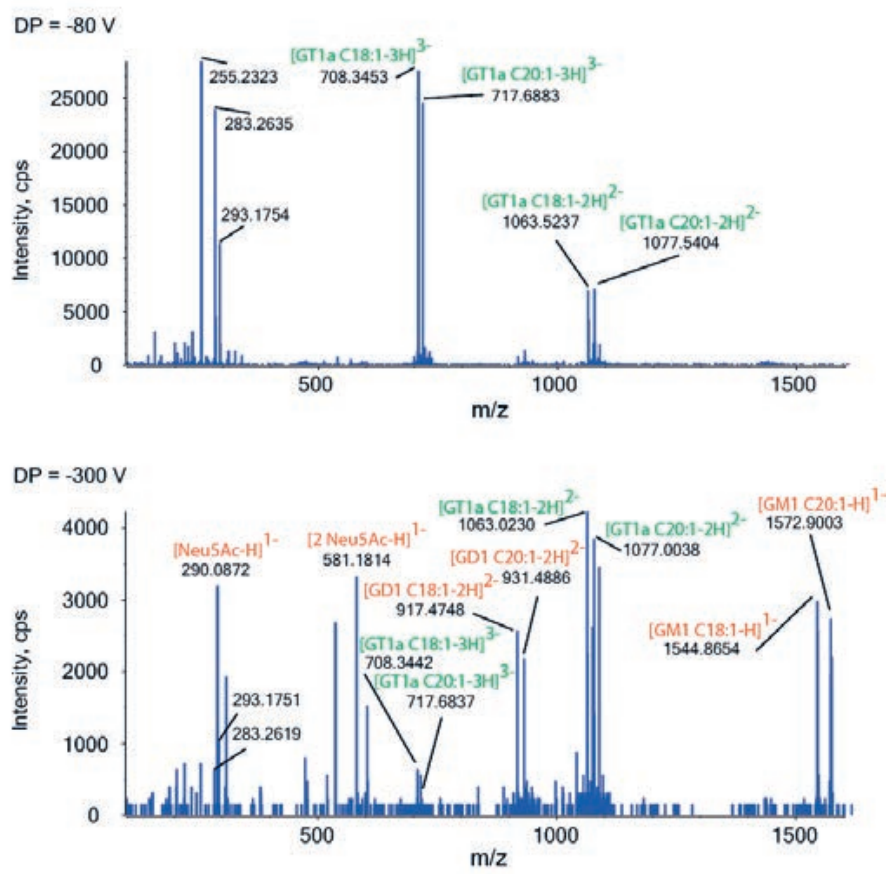

Fig. 5. Effect of the electrospray interface declustering potential on the analysis of GT1a: Appropriate setting of the potential enables the detection of the doubly and triply charged intact gangliosides (indicated in green color in the upper panel). Elevated potential results in dissociation of monomeric and dimeric sialic acids of GT1a and gives rise to fragment ions mimicking different gangliosides not present in the sample such as the doubly charged GD1 and singly charged GM1 (indicated in orange color in the lower panel).

The conversion of one ganglioside into another by the loss of sialic acids is to be avoided while simultaneously maintaining good ionization efficiency and analyte ion desolvatization.
Therefore, careful balancing of the ion source parameters is crucial for simultaneously gaining a high reliability of compound identification and strong signal intensity in ganglioside analysis by LC-MS.

\section{Conclusions}

In this study, a set of strongly amphiphilic gangliosides was successfully separated by HILIC, including the chromatographically demanding disialo-ganglioside isomers GD1a and GD1b. Tandem mass spectrometric characterization of the separated gangliosides was performed by information-dependent acquisition employing electrospray ionization in the negative ionization mode. Fragment ions indicative for the carbohydrate head groups of the individual gangliosides could be identified, allowing a clear structural identification, including the information about monoand disialic structural features. Results demonstrate the importance of optimized ion source parameters, including ion source gas temperature and declustering potential, in order to avert a deceptive ganglioside transformation by sialic acid cleavage.

\section{Acknowledgements}

We thank BÜHLMANN Laboratories AG (Schönenbuch, Switzerland) for generously providing ganglioside samples.

\section{Received: October 31, 2021}

[1] R. K. Yu, Y.-T. Tsai, T. Ariga, M. Yanagisawa, J. Oleo Sci. 2011, 60, 537, https://doi.org/10.5650/jos.60.537.

[2] R. K. Yu, T. Ariga, M. Yanagisawa, G. Zeng, in 'Glycoscience: Chemistry and Chemical Biology', Eds. B. O. Fraser-Reid, K. Tatsuta, J. Thiem, Springer Berlin Heidelberg, Berlin, Heidelberg, 2008, pp. 1671, https://doi.org/10.1007/978-3-540-30429-6_41.

[3] E. Klenk, Biol. Chem. 1942, 273, 76, https://doi.org/10.1515/bchm2.1942.273.1-2.76.

[4] S. Sipione, J. Monyror, D. Galleguillos, N. Steinberg, V. Kadam, Front. Neurosci. 2020, 14, 572965, https://doi.org/10.3389/fnins.2020.572965.

[5] H. J. Willison, B. C. Jacobs, P. A. van Doorn, Lancet 2016, 388, 717, https://doi.org/10.1016/S0140-6736(16)00339-1.

[6] R. L. Schnaar, in 'Sialic Acids, Part II: Biological and Biomedical Aspects', Vol. 76, Ed. D. C. B. T.-A. in C. C. and B. Baker, Academic Press, 2019, pp. 113, https://doi.org/https://doi.org/10.1016/bs.accb.2018.09.002.

[7] T. Maula, I. Artetxe, P.-M. Grandell, J. P. Slotte, Biophys. J. 2012, 103, 1870, https://doi.org/10.1016/j.bpj.2012.09.018.

[8] T. Kolter, ISRN Biochem. 2012, 2012, 506160, https://doi.org/10.5402/2012/506160.

[9] S. Sonnino, A. Prinetti, 'Gangliosides: Methods and Protocols', Springer New York, 2018.

[10] X. Zhang, F. L. Kiechle, Ann. Clin. Lab. Sci. 2004, 34, 3.

[11] K. Ikeda, T. Shimizu, R. Taguchi, J. Lipid Res. 2008, 49, 2678 , https://doi.org/10.1194/jlr.D800038-JLR200.

[12] K. Ikeda, R. Taguchi, Rapid Commun. Mass Spectrom. 2010, 24, 2957, https://doi.org/10.1002/rcm.4716.

[13] R. C. Barrientos, Q. Zhang, Anal. Chem. 2018, 90, 2578, https://doi.org/10.1021/acs.analchem.7b04044.

[14] J. Lee, H. Hwang, S. Kim, J. Hwang, J. Yoon, D. Yin, S. Il Choi, Y.-H. Kim, Y.-S. Kim, H. J. An, Cells 2019, 8, 1323, https://doi.org/10.3390/cells8111323.

[15] A. L. P. Gobburi, E. W. Kipruto, D. M. Inman, D. J. Anderson, J. Liq. Chromatogr. Relat. Technol. 2021, 44, 114, https://doi.org/10.1080/10826076.2020.1856136.

[16] T. Hu, Z. Jia, J.-L. Zhang, Anal. Chem. 2017, 89, 7808, https://doi.org/10.1021/acs.analchem.7b02023.

[17] X.-Y. Meng, L.-F. Yau, H. Huang, W.-H. Chan, P. Luo, L. Chen, T.-T. Tong, J.-N. Mi, Z. Yang, Z.-H. Jiang, J.-R. Wang, Chem. Phys. Lipids 2019, 225, 104813, https://doi.org/10.1016/j.chemphyslip.2019.104813.

[18] B. Buszewski, S. Noga, Anal. Bioanal. Chem. 2012, 402, 231, https://doi.org/10.1007/s00216-011-5308-5.

[19] D. V McCalley, J. Chromatogr. A 2017, 1523,49, https://doi.org/10.1016/j.chroma.2017.06.026.

[20] D.-Q. Tang, L. Zou, X.-X. Yin, C. N. Ong, Mass Spectrom. Rev. 2016, 35, 574, https://doi.org/10.1002/mas.21445.

[21] R. Pérez-Míguez, M. Castro-Puyana, E. Sánchez-López, M. Plaza, M. L. Marina, Molecules 2020, 25, https://doi.org/10.3390/molecules25040887.

[22] R. Hájek, R. Jirásko, M. Lísa, E. Cífková, M. Holčapek, Anal. Chem. 2017, 89, 12425, https://doi.org/10.1021/acs.analchem.7b03523. 
[23] R. Hájek, M. Lísa, M. Khalikova, R. Jirásko, E. Cífková, V. Student Jr, D. Vrána, L. Opálka, K. Vávrová, M. Matzenauer, B. Melichar, M. Holčapek, Anal. Bioanal. Chem. 2018, 410, 1, https://doi.org/10.1007/s00216-018-1263-8.

[24] D. Olešová, P. Majerová, R. Hájek, J. Piešt’anský, R. Brumarová, A. Michalicová, B. Jurkanin, D. Friedecký, A. Kováč, Int. J. Mol. Sci. 2021, 22 https://doi.org/10.3390/ijms222212581.

[25] Y. Ma, X. Wang, Z. Wang, P. Cong, J. Xu, C. Xue, J. Agric. Food Chem. 2021, 69, 7641, https://doi.org/10.1021/acs.jafc.1c02058.

[26] X. Wang, X. Wang, P. Cong, X. Zhang, H. Zhang, C. Xue, J. Xu, Food Chem. 2021, 352, 129379, https://doi.org/https://doi.org/10.1016/j.foodchem.2021.129379.

[27] A. J. Alpert, J. Chromatogr. A 1990, 499, 177, https://doi.org/https://doi.org/10.1016/S0021-9673(00)96972-3.

[28] B. Fong, C. Norris, E. Lowe, P. McJarrow, Lipids 2009, 44, 867, https://doi.org/10.1007/s11745-009-3327-1.
[29] M. Rožman, J. Am. Soc. Mass Spectrom. 2016, 27, 91 , https://doi.org/10.1007/s13361-015-1247-2.

\section{License and Terms}

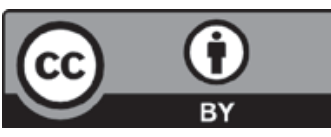

This is an Open Access article under the terms of the Creative Commons Attribution License CC BY 4.0. The material may not be used for commercial purposes.

The license is subject to the CHIMIA terms and conditions: (https://chimia.ch/chimia/about).

The definitive version of this article is the electronic one that can be found at https://doi.org/10.2533/chimia.2022.109 